\title{
COMPUTER VISION APPLIED TO ROAD LINES RECOGNITION USING MACHINE LEARNING
}

\author{
C. H. Rodríguez-Garavito, A. Ponz, F. García, A. de la Escalera and J.M. Armingol. \\ Intelligent Systems Lab. \\ Universidad Carlos III de Madrid, UC3M. \\ Madrid, Spain. \\ cesarhernan.rodriguez@alumnos.uc3m.es , \{apv, fegarcia, escalera, armingol \}@ing.ucm3.es \\ C. H. Rodríguez-Garavito. \\ Automation engineering department. \\ Universidad de La Salle, Bogotá Colombia. \\ cerodriguez@unisalle.edu.co
}

\begin{abstract}
According to the Department for Transport statistics in UK, around $\mathbf{1 0 0 . 0 0 0}$ accidents were reported in 2013 [13], and almost $25 \%$ of them were related to impairment or distraction factors. Advanced Driver Assistance Systems (ADAS) are a powerful tool for road safety that can help to mitigate this problem. This paper presents a robust road lane detection and classification algorithm, one of the most important tasks in ADAS. This paper describes a road line detection algorithm based on a segmentation algorithm designed according to the constraints defined in the legal regulation for road marks. Later, pairs of lines, separated a fixed distance, are searched in the bird view of the road image. The bird view transformation is applied to the captured images, using the extrinsic parameters estimation algorithm reported in [10]. After the extraction of the road lines profiles, they are characterized using a specifically designed descriptor based on both space and frequency values. The descriptors are used in the supervised training of a Support Vector Machines classifier, whose performance is compared against the previous version of the module, a heuristic based approach. The performed tests showed a considerable increase of the system performance using the SVM approach, in comparison with the previous heuristic approach.
\end{abstract}

\section{Keywords—Road Line Segmentation; Road Line Classification; Support Vector Machine, Bird Eye View}

\section{INTRODUCTION}

Nowadays, interest in Advanced Driving Assistance Systems (ADAS) is growing due to their contribution to effectively reduce human error related accidents; therefore, they have become a differentiating element in the automotive market.

One of the key points in ADAS applications are automatic and intelligent algorithms and strategies such as machine learning. Among the multiple tasks that might take advantage of these useful techniques are: Detection and identification of road signs [1], vehicle [2] and pedestrian [3] detection, estimation of driver distraction [4], and environment interpretation and understanding [5].

The present work is focus on the extraction and detection of road lane delimitation lines. This task must be reliable in order to build a complete model of the road environment, that is, determine the type of road, number of lanes, type of lanes, and vehicle position with reference to the current lane, among many other characteristics.

Several works have already addressed this issue on the basis of different techniques with promising results: in [6] road lines are classified as solid or dashed, by analyzing the gaps between measurement points, which are predicted throughout a projected model lane and a complex kalman filter. In [7], line boundaries detection is performed based on a priori information about its position in the processed image; a ROI at the initial position of each boundary is used to get a statistic intensity model and built a binary path for each line. Next, a cascade classifier is applied, and the sub-classifiers are tuned for dashed, dashed-solid, solid-dashed, single-solid and doublesolid line patterns, they are based on measure like fraction of marking-related pixels and temporal autocorrelation. Another approach using off-line information of the road curvature is proposed in [8], where a priori information, together with external sensors measurements from GPS and gyroscope, allow tracking model estimation by the use of an AMF (Approximated Median Filter).

This paper presents the Road Lane Classification module of the IvvI 2.0 project (Intelligent Vehicle based on Visual Information) [12]. Its goal is to automatically detect the position, type, and number of the road lanes with a stereo onboard camera inside of the Intelligent System Lab at Carlos III University experimental vehicle. In this work, three types of lane boundaries are considered, namely: solid, dashed and 
merge, using classification by heuristic classifier [9] and support vector machine, SVM classifier.

The article is divided into the following sections: Section II explains how lane boundary lines are segmented. Section III focuses on the algorithms for line description and classification. Section IV describes the performance tests executed for the classifiers, and Section V presents some conclusions for the work.

\section{SEGMENTATION OF IN-ROAD LANE SEPARATOR LINES}

The process starts with the capture of a pair of stereo images, from where a three-dimensional representation of the road environment is built. This representation is called Point Cloud (PC), and is a collection of points in the space referred to a system of coordinates located in the reference camera of the stereo pair. As described in [10], it is possible to obtain the parametric definition of the most populated plane in the PC. Some information obtained from this plane determines the spatial position of the camera that records the images. The parameters that define this spatial position are called "extrinsic camera parameters", and together with the intrinsic camera parameters, that is, sensor and optical system specifications, allows the conversion of the actual perspective of the captured scene into a more convenient perspective for road lines detection. In this new perspective, called "bird view perspective" or "upper view", the parallel geometry between road lines in the real world is visually preserved. This perspective transformation or homograph is shown in Equation $\square \square \square$

$$
\mathrm{s} *{ }^{\mathrm{c}} \mathrm{P}={ }^{\mathrm{c}} \mathrm{P}_{\mathrm{s}}={ }^{\mathrm{c}} \mathrm{H}_{\mathrm{bv}}{ }^{\mathrm{bv}} \mathrm{P}
$$

where a relation is established between every pixel position it the bird-view ${ }^{b v} \mathrm{P}_{\mathrm{i}}$ and pixels ${ }^{c} \mathrm{P}_{\mathrm{i}}$ in the original image, $\mathrm{s}$ is an scaling factor which appears as the third component of the point resulting from the homographic transformation, ${ }^{c} \mathrm{P}_{\mathrm{S}}$.

The matrix ${ }^{c} \mathrm{H}_{\mathrm{bv}}$ is decomposed as seen in Equation (2), details are shown in Table 1.

$$
{ }^{\mathrm{c}} \mathrm{H}_{\mathrm{bv}}=\mathrm{K} *{ }^{\mathrm{c}} \mathrm{T}_{\mathrm{m}} *{ }^{\mathrm{m}} \mathrm{T}_{\mathrm{bv}} * \mathrm{~K}^{-1}
$$

TABLE I. PARAMETRIC DESCRIPTION OF A HOMOGRAPHIC TRANSFORMATION.

\begin{tabular}{|c|c|}
\hline $\begin{array}{c}\text { Parametric } \\
\text { Matrix }\end{array}$ & Meaning \\
\hline$K$ & Intrinsics matrix \\
\hline${ }^{c} T_{m}$ & $\begin{array}{c}\text { Spatial transformation from road plane into } \\
\text { camera position }\end{array}$ \\
\hline${ }^{m} T_{b v}$ & $\begin{array}{c}\text { Spatial transformation from bird view } \\
\text { position into road plane }\end{array}$ \\
\hline
\end{tabular}

The result of the transformation can be seen in Fig. 1.

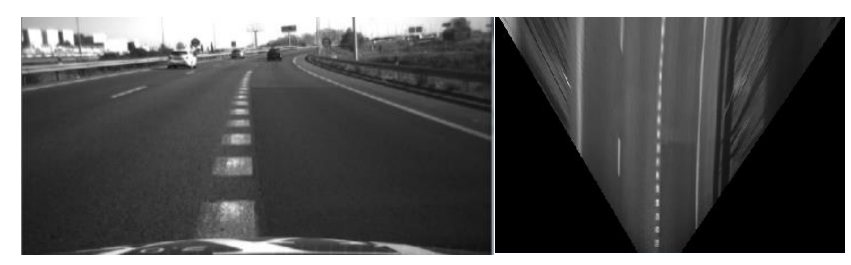

Fig. 1. Homographic transformation from road image into bird view and road lines detection.

After the homographic transformation, a detection of the pixels meeting the characteristics of a road line is performed. For the present approach, these characteristics were defined on the basis of variations of the intensity gradient, direction gradient, and the road line width, in order to create a road line mask as shown in Fig, 2.

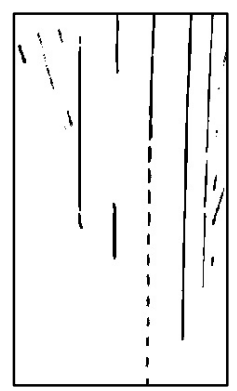

Fig. 2. Road line mask in bird view perspective.

\section{A. Road lines and lanes detection}

Using the Hough Transform, line detection is performed in the bird view image over road line mask. The direction of the lines belonging to the road is not known a priori, so the assumption that the angle $\theta_{\mathrm{R}}$ of the searched lanes will match the angle with the highest magnitude in a histogram of orientations computed over the total of detected lines will be made.

From the set of detected lines, the pairs of lines separated a specific distance ( i.e. lade width \pm road mark width threshold) parallel to each other and whose orientation matches the road angle, $\theta_{R}$, are identified. These characteristic were based on Spain's IC 8.2 standard [11]. The filtered lanes are not necessarily consecutive, but may be overlapping as shown in Fig. 3.

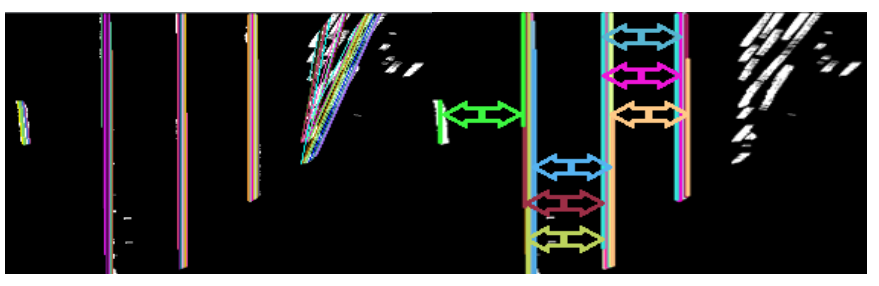

Fig. 3. Lines detected with Hough Transform and non-consecutive lanes segmented.

\section{B. Identification of lanes on the road in adjacent fashion}

In order to create a spatially ordered structure, i.e. a road with consecutive lanes, the detected lanes are chained using a 
separating line identifier, thus it is possible to find their order in the road.

Two successive ordering processes are performed. A first ordering is made, starting at a random lane. Initially, one of the two directions, $\theta_{\mathrm{R}}+/-\pi$ in which adjacent lanes are labeled from an initial lane. Once the search in one direction is finished, a new search is performed, starting from the first lane in the opposite direction.

The lanes obtained from the search are stored in consecutive order. Each one is composed of a pair of lines matching the aforementioned separation constraints. Information from common boundaries is fused and unique lines between lanes are created as shown in Fig. 4.

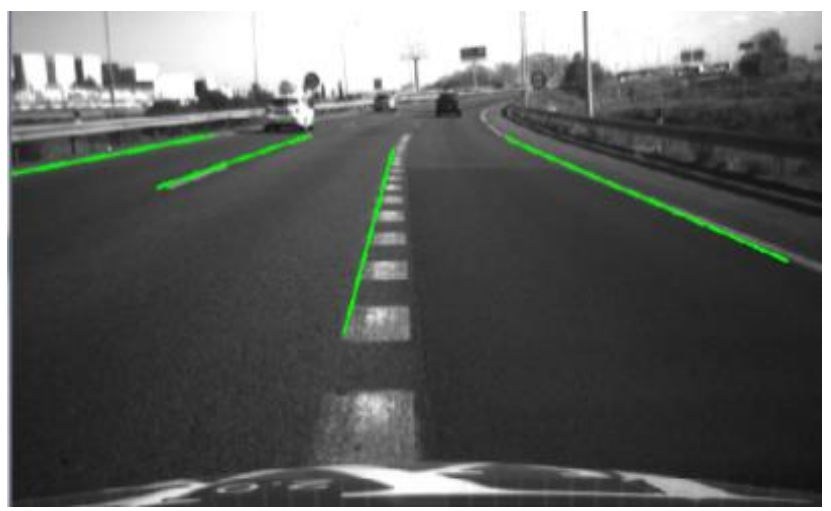

Fig. 4. Lane Boundaries defined by fusing spatially adjacenct lanes.

\section{LINES EXTRACTION AND CLASSIFICATION}

Road lines classification starts with the extraction of detected line profiles, $f(p i x)$. Profiles are vectors of binary values whose size matches the length of the detected line. The array is initialized by scanning the positions of the detected line on the road lines mask. An example of a detected line profile can be seen in Fig. 5 .
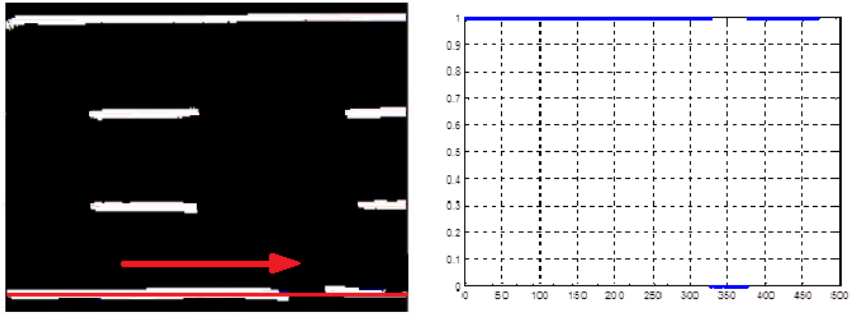

Fig. 5. Detected line profile, $f(p i x)$.

The detected lines are obtained from the road lines mask, which may contain noise and spurious elements. Thus, a fuzzy logic strategy called center of gravity, is employed in order to defuzzify an end-of-line profile into a corrected concrete value.

\section{Adjustment by center of gravity}

The position of each end-of-line is corrected by computing the center of gravity of its profile, which is taken in the direction perpendicular to the line checked, in a neighborhood of two road line widths, as depicted in Fig. 6.
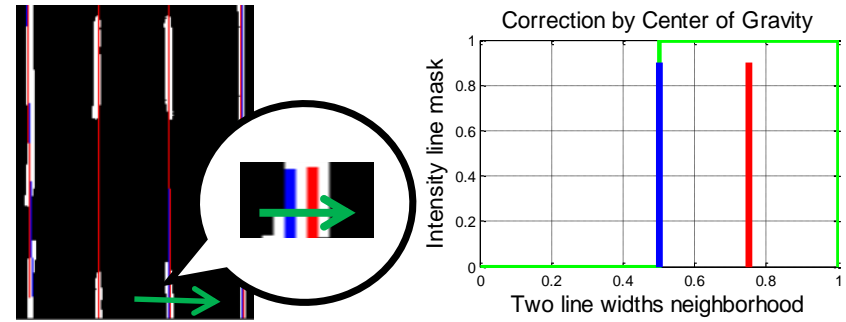

Fig. 6. Correction by center of gravity of the end of line coordinates for each lane in the road ordered structure.

\section{Descriptor}

The next step in the machine learning based strategy for road lines classification is the unequivocal description of the elements to classify, that is, to find a representation that unambiguously identifies all the possible elements to classify. Two different elements can't share the same descriptor; furthermore, the descriptors must be as small as possible in order to allow efficient training and prediction processes.

The descriptor chosen in this case is based on a mix of features in both spatial and frequency domain. The two first features are the mean value of the line profile, and its length,in meters. The remaining features correspond to the frequencies of the $\mathrm{n}$ first power peaks, in descendent order. The selected features take into account the difference in the spatial frequency representation of the different line types, as seen in Fig. 7.

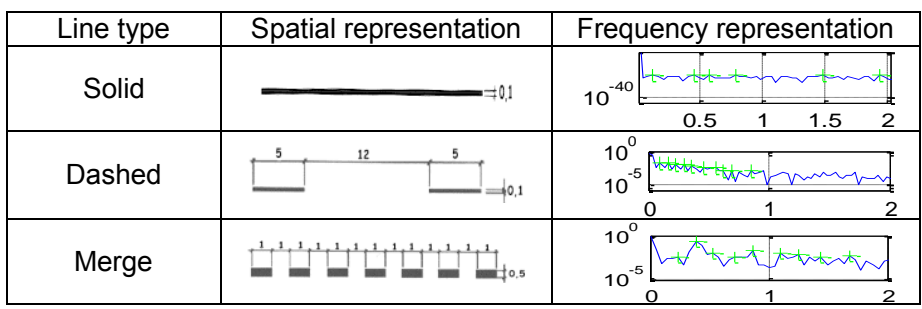

Fig. 7. Line profiles characterization in spatial and frequency domains.

Fig. 8 shows the power spectrum $F(f(p i x))$ for a detected line, $f(p i x)$. Two different methods were developed for line classification: A heuristic method, based on the behavior of the intensity peaks in the descriptor, and a second method based on Support Vector Machines, a classification machine learning algorithm.
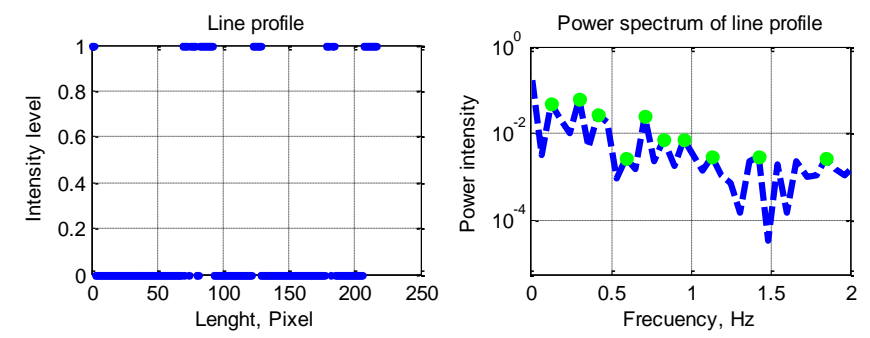

Fig. 8. Power spectrum $F$, for a detected line, $f($ pix $)$. 


\section{E. Heuristic classifier}

In the case of the heuristic classifier, the decision rules are obtained by analyzing a great collection of sampled lines, along with their corresponding space and frequency spectrum, as shown in Fig. 9 and Fig. 10.

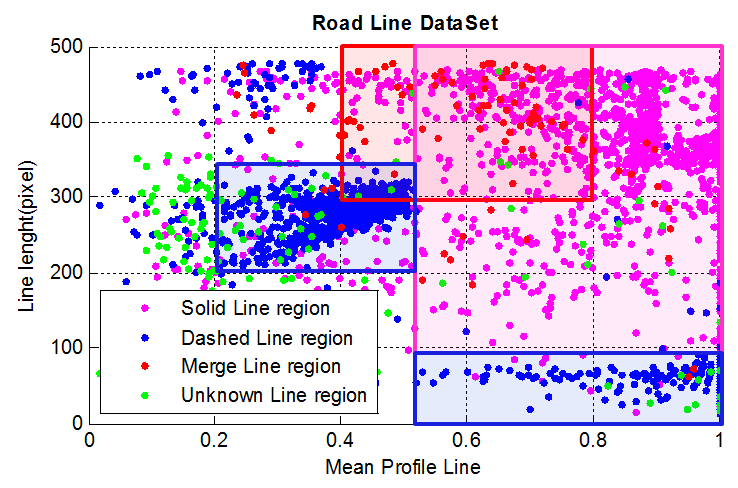

Fig. 9. Feacture Space for descriptor lines.

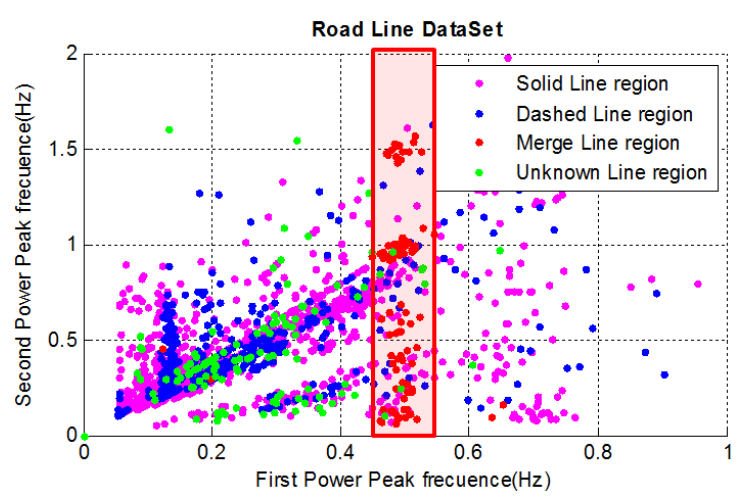

Fig. 10. Feacture frecuency for descriptor lines.

The decision algorithm is a sequence of three binary classifiers, $\mathrm{C}_{1}, \mathrm{C}_{2} \mathrm{y} \mathrm{C}_{3}$. shown in Fig. 11 .

- $\omega_{1}$ : The distance from $0.5 \mathrm{~Hz}$ to some of the 3 first peaks of $\mathrm{F}$ is smaller than $0.05 \mathrm{~Hz}$ ?

- $\omega_{2}$ : The mean of $\mathrm{f}$ is greater than 0.4 and smaller than 0.8 ?

- $\omega_{3}$ : The length of $f$ is greater than the three lengths of a dashed line segment?

- $\omega_{4}$ : The mean of $\mathrm{f}$ is greater than 0.52 ?

- $\omega_{5}$ : The length of $\mathrm{f}$ is greater than the length of a dashed line segment?

- $\omega_{6}$ : The mean of $f$ is greater than 0.2 and smaller than 0.52 ?

- $\omega_{7}$ : The length of $\mathrm{f}$ is greater than the 2 lengths of a dashed line segment and smaller than 3.3 lengths of a dashed line segment?
- $\omega_{8}$ : The length of $\mathrm{f}$ is smaller than the length of a dashed line segment?

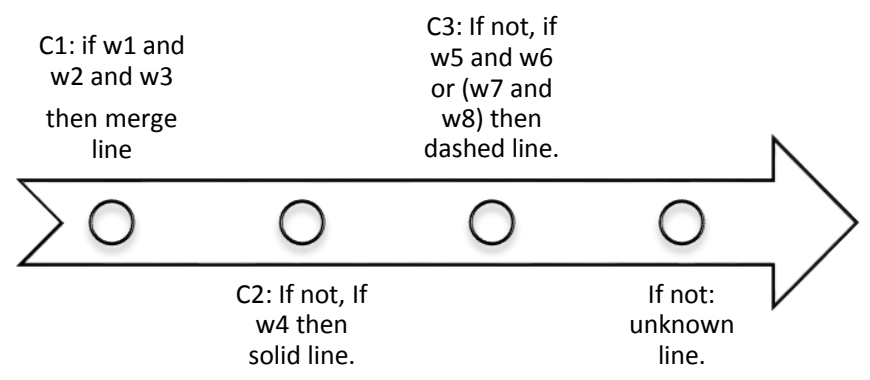

Fig. 11. Decision algorithm for heuristic classification.

\section{F. SVM classifier}

SVM, the machine learning based method, uses the same descriptor, and associates each line type to a different class. The learning and prediction approaches are shown in Fig. 12.

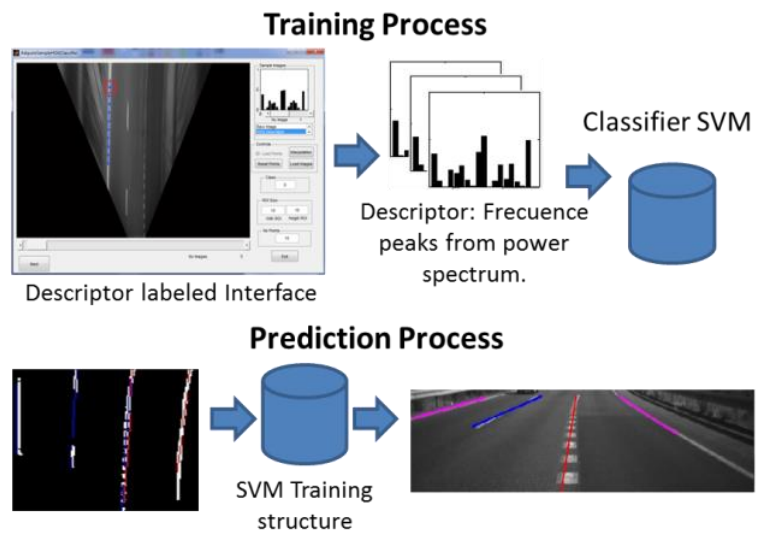

Fig. 12. Stages of the machine learning classification process.

Two different processes were necessary to develop the SVM classification algorithm. The first one refers to the database creation, populated with an adequate amount of manually labeled descriptors, some of them are later used as a ground truth. This information database also provides the supervised learning examples that iteratively adjust an optimal parametric SVM structure. The classifier is then implemented using a radial base kernel and a multiclass strategy. Four binary classifiers are trained, one per class.

After the training process, the trained SVM structures are stored for recovery in the prediction stage of new non-trained descriptors. The detection is made by checking how close is an input descriptor to each classification region given by a different classifier. The closest region to the descriptor determines the selected class. A visual representation of the classification regions, using line profile mean and first power peak frequency as classification features, is shown in Fig. 13. 


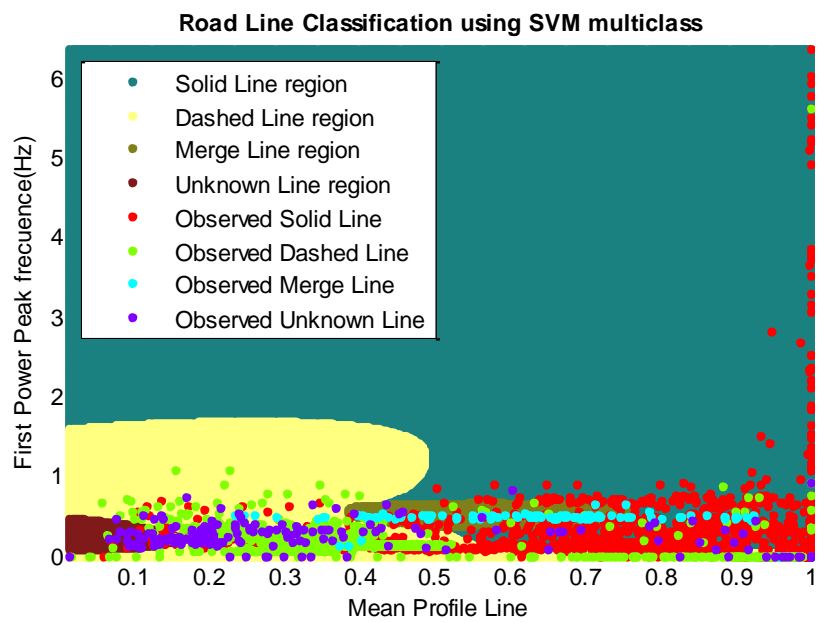

Fig. 13. Road lines classification using multiclass SVM.

\section{TESTS AND RESULTS}

Tests of the road lines classifiers are made over a database of 1000 images. Based on them, a collection of 4100 manually labeled descriptor-class pairs were made. $80 \%$ of data was used for training and $20 \%$ of data was used for validation.

Table 2 depicts the confusion matrix associated to each classification process with SVM descriptor. Table 3 shows the confusion matrix associated to heuristic classification. These are the results of prediction versus ground truth comparisons over the validation data.

TABLE II. CONFUSION MATRIX SVM CLASSIFIER.

\begin{tabular}{|c|c|c|c|c|}
\hline & Solid & Dashed & Merge & Unkown \\
\hline Solid & 298 & 24 & 6 & 13 \\
\hline Dashed & 17 & 375 & 0 & 3 \\
\hline Merge & 5 & 2 & 25 & 0 \\
\hline Unkown & 7 & 10 & 1 & 19 \\
\hline
\end{tabular}

TABLE III. CONFUSION MATRIX HEURISTIC CLASSIFIER.

\begin{tabular}{|c|c|c|c|c|}
\hline & Solid & Dashed & Merge & Unkown \\
\hline Solid & 193 & 78 & 40 & 30 \\
\hline Dashed & 3 & 334 & 13 & 45 \\
\hline Merge & 2 & 2 & 16 & 12 \\
\hline Unkown & 2 & 6 & 5 & 24 \\
\hline
\end{tabular}

Finally, based on the classification results, classification methods performances can be measured using percentage of true positives per class, as shown in Fig. 12. and Table 4.

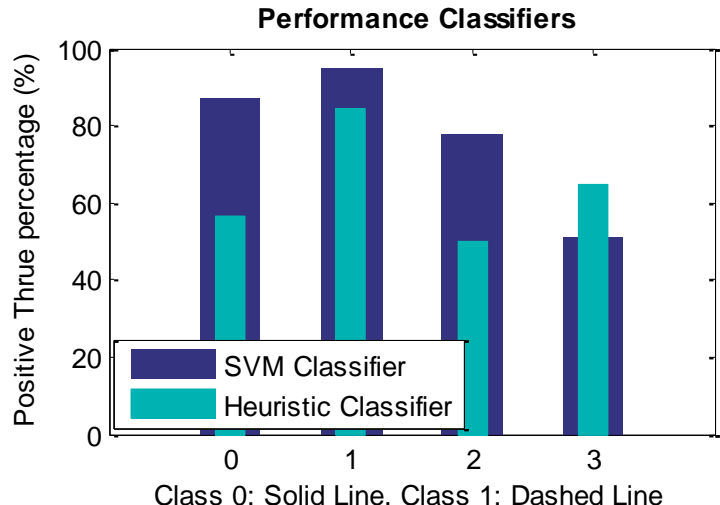

Class 2: Merge Line, Class 3: Line Unknown

Fig. 14. Performance of the analized classifiers: Heuristic and SVM.

TABLE IV. Percentage ReCognition Line Class by CALSSIFICATION METHOD.

\begin{tabular}{|c|c|c|c|c|}
\hline \multirow{2}{*}{ Method } & \multicolumn{4}{|c|}{ Percentage Recognition Line Class } \\
\cline { 2 - 5 } & Solid & Dashed & Merge & Unknown \\
\hline Heuristic & 0.566 & 0.8456 & 0.5 & 0.6486 \\
\hline SVM & 0.8739 & 0.9494 & 0.7813 & 0.5135 \\
\hline
\end{tabular}

\section{CONCLUSIONS}

An improved version of the IvvI 2.0 road lines detection and classification module is presented. The road lanes segmentation procedure shown has proven to be a robust method, invariant both to high shapes changes and to occlusions. This segmentation stage is appropriate for further lane tracking.

The main stage of the work, that is, road line classification using SVM over a 1000 images collection, shows a significant improvement in true positive detections. Success rates increased a $31 \%$ for solid lines, $10 \%$ for dashed lines, and $28 \%$ for merge lines. This classification strategy is a systematic method that allows for the inclusion of further images characteristics, such as changes in illumination conditions or new road signs.

The remarkable detection line results obtained in the aforementioned tests were achieved based on single frame detection. Further steps will be focused on developing a tracking method that will help to overcome difficult situations and exceptional misclassifications by adding time consistency.

\section{ACKNOWLEDGMENTS}

This work was supported by automation engineering department from de La Salle University, Bogotá-Colombia; Administrative Department of Science, Technology and Innovation (COLCIENCIAS), Bogotá-Colombia and the Spanish Government through the CICYT projects (TRA201348314-C3-1-R) and (TRA2011-29454-C03-02) and Comunidad de Madrid through SEGVAUTO-TRIES (S2013/MIT-2713). 


\section{REFERENCES}

[1] Maldonado-Bascon, S., Lafuente-Arroyo, S., Gil-Jimenez, P., GomezMoreno, H., \& Lopez-Ferreras, F. (2007). Road-sign detection and recognition based on support vector machines. Intelligent Transportation Systems, IEEE Transactions On, 8(2), 264-278. doi:10.1109/TITS.2007.895311

[2] Zezhi Chen, Pears, N., Freeman, M., \& Austin, J. (2009). Road vehicle classification using support vector machines. Intelligent Computing and Intelligent Systems, 2009. ICIS 2009. IEEE International Conference On, , 4 214-218. doi:10.1109/ICICISYS.2009.5357707

[3] Olmeda, D., de la Escalera, A., \& Armingol, J. M. (2009). Detection and tracking of pedestrians in infrared images. Signals, Circuits and Systems (SCS), 2009 3rd International Conference On, 1-6. doi:10.1109/ICSCS.2009.5412297

[4] Yulan Liang, Reyes, M. L., \& Lee, J. D. (2007). Real-time detection of driver cognitive distraction using support vector machines. Intelligent Transportation Systems, IEEE Transactions On, 8(2), 340-350. doi:10.1109/TITS.2007.895298

[5] Zhang, H., Hou, D., \& Zhou, Z. (2005). A novel lane detection algorithm based on support vector machine. Progress in Electromagnetics Research Symposium, Hangzhou, China,

[6] Risack, R., Klausmann, P., Krüger, W., \& Enkelmann, W. (1998). Robust lane recognition embedded in a real-time driver assistance system. In Proc. IEEE IV,

[7] Paula, M. B. d., \& Jung, C. R. (2013). Real-time detection and classification of road lane markings. Graphics, Patterns and Images (SIBGRAPI), 2013 26th SIBGRAPI-Conference On, 83-90.

[8] Maeda, T., Hu, Z., Wang, C., \& Uchimura, K. (2008). High-speed lane detection for road geometry estimation and vehicle localization. SICE Annual Conference, 2008, 860-865.

[9] Collado, J. M., Hilario, C., De La Escalera, A., \& Armingol, J. M. (2006). Adaptative road lanes detection and classification. Advanced Concepts for Intelligent Vision Systems, 1151-1162.

[10] Rodriguez-Garavito, C., Ponz, A., Garcia, F., Martín, D., de la Escalera, A., \& Armingol, J. (2014). Automatic laser and camera extrinsic calibration for data fusion using road plane. Information Fusion (FUSION), 2014 17th International Conference On, 1-6.

[11] Espanya., D. G. d. C. (1991). Marcas viales : Norma de carreteras 8.2IC. Madrid: MOPU. Secretaría General Técnica. Centro de Publicaciones.

[12] Martín, D., García, F., Musleh, B., Olmeda, D., Peláez, G., Marín, P., Armingol, J. M. (2014). IVVI 2.0: An intelligent vehicle based on computational perception. Expert Systems with Applications, 41(17), 7927-7944. doi:http://dx.doi.org/10.1016/j.eswa.2014.07.002

[13] DfT. (2013). Reported road casualties Great Britain: 2013. Annual Report. London: The Stationery Office. 